\title{
Corela
}

Cognition, représentation, langage

HS-1 1 | 2012

RJC Cotexte, contexte, situation

\section{Le pluriel en fang ntumu}

\section{Régis Ollomo Ella}

\section{OpenEdition}

\section{Journals}

Édition électronique

URL : http://journals.openedition.org/corela/2520

DOI : $10.4000 /$ corela. 2520

ISSN : 1638-573X

\section{Éditeur}

Cercle linguistique du Centre et de l'Ouest - CerLICO

\section{Référence électronique}

Régis Ollomo Ella, «Le pluriel en fang ntumu », Corela [En ligne], HS-11 | 2012, mis en ligne le 02 avril 2012, consulté le 30 avril 2019. URL : http://journals.openedition.org/corela/2520 ; DOI : 10.4000/ corela. 2520

Ce document a été généré automatiquement le 30 avril 2019.

\section{(c) (i) (-)}

Corela - cognition, représentation, langage est mis à disposition selon les termes de la licence Creative Commons Attribution - Pas d'Utilisation Commerciale - Partage dans les Mêmes Conditions 4.0 International. 


\title{
Le pluriel en fang ntumu
}

\author{
Régis Ollomo Ella
}

\section{Introduction}

1 Le terme ntúmú désigne à la fois une variante dialectale de la langue fang, et les locuteurs de ce dialecte.

2 Le fang une langue bantu parlée au Cameroun, au Congo, au Gabon et en GuinéeEquatoriale. Dans la classification de Guthrie, il appartient au groupe Yaoundé-Fang (Ewondo-Fang) (A70) et il porte l'indice A75. Autrement dit c'est la cinquième langue du groupe 7 de la zone A. Le ntúmú porte pour sa part l'indice A75A.

3 Du point de vue de la communication, il faut signaler qu'il existe une intercompréhension totale aussi bien entre les cinq dialectes fang (ntumu, okak, mvén, nzaman, atsi), qu'entre le fang et certaines langues du groupe A70, notamment le bulu et l'éwondo parlés au Cameroun.

4 Les locuteurs ntúmú sont localisés au nord Gabon, au sud Cameroun et en GuinéeEquatoriale ( $\mathrm{Cf}$ : carte1). Les données sur lesquelles se base cette étude ont été collectées en 1997 au sud Cameroun, dans la localité de Mvi-Mangale par Marie Françoise ROMBI.

5 Comme l'ensemble des langues bantu, le fang procède à la classification de ses nominaux. Les nominaux d'une même classe régissent les mêmes accords et la langue leur affecte généralement un même indice préfixal dont la forme varie en fonction du contexte phonétique : c'est le préfixe nominal (PN). Il peut être à signifiant zéro |ø-|, de type C-, V-, ou CV-.

6 Le fang ntúmú est une langue tonale à deux ou trois registres (haut, moyen, bas), et accentuelle sur la première syllabe de la base (racine ou thème nominal).

7 Le préfixe nominal apporte des informations non seulement sur la classe du nominal, mais également sur le nombre. Il existe donc des préfixes nominaux de type singulier et de type pluriel. 
Une observation de certains nominaux pluriel en fang ntúmú, notamment ceux ayant une nasale comme préfixe nominal au singulier, semble montrer que le pluriel s'obtient par l'agencement de deux préfixes : celui du pluriel suivi de celui du singulier. Dès lors se pose le problème d'une éventuelle double préfixation en fang ntúmú, problème dont traitera en grande partie le texte qui va suivre.

Nous démontrerons tout au long de ce travail que le pluriel en fang ntúmú résulte, non pas d'un processus de double préfixation, comme certains travaux le laissent entendre, mais plutôt d'une construction préfixale complexe de type CVN.

Carte1 : localisation du fang ntúmú

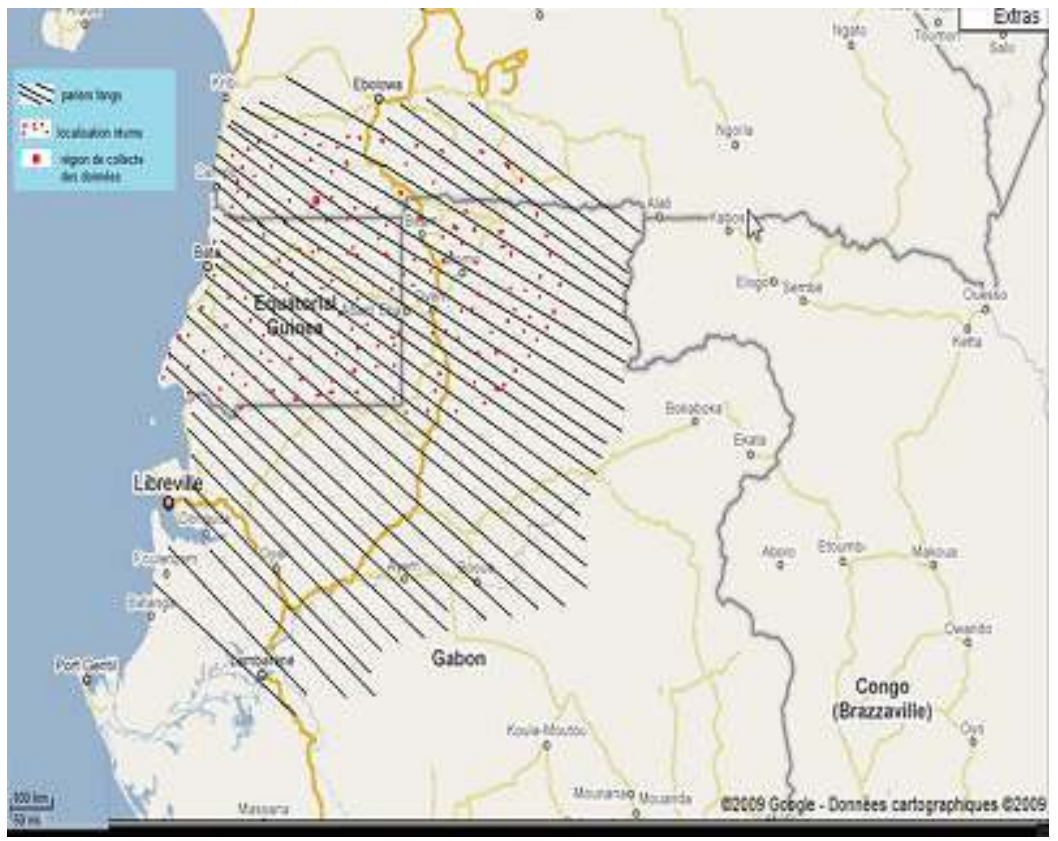

Source : Régis OLLOMO ELLA sur fond de carte Google maps.

\section{La morphologie nominale}

\subsection{Généralités}

Dans les langues bantu, la structure du nominal est généralement de type PN+BASE. La base peut être de type nominale (thème nominal indécomposable), ou verbo-nominale (racine) :

Exemples :

1) èlé "arbre" |è-lé| /PN7- Thème (arbre)/

2) bilé "arbres" |bi-lé| /PN8- Thème (arbre)/

3) ádzi "manger" |á-dzí|/PV-RAC (manger)/

4) èdzíjà "complément alimentaire" |è-dzí-jà| /PN7-RAC (manger)-suffixe (accompagnement)/

Les racines verbo-nominales permettent d'obtenir des verbes lorsqu'elles sont associées au préfixe verbal |a-| (qui est en réalité le préfixe de classe 5), et des noms lorsqu'elles sont associées à un préfixe nominal. 
12 Dans l'exemple 2, le préfixe nominal de classe 8 est de type pluriel. Il ne pose aucun problème d'identification ni de découpage morphologique.

\subsection{Les préfixes nominaux en fang}

Le tableau ci-dessous, proposé par MBA NKOGHE, présente un récapitulatif des préfixes nominaux du fang ainsi que les différents appariements singulier/pluriel.

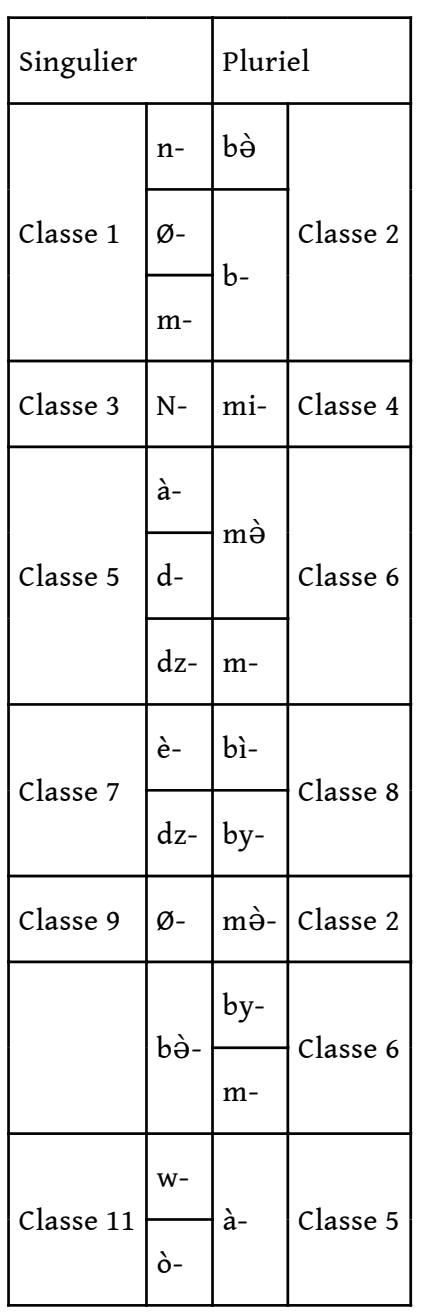

\subsection{La problématique du ntúmú}

Certains nominaux fang ntúmú comportent, au singulier, une nasale syllabique homorganique de la consonne suivante (N-). Celle-ci joue le rôle d'indice de classe. C'est le cas des préfixes nominaux des classes 1et 3.

Exemples :

5) Cl.1/2 : ǹtòbò "habitant" / bòtòbò "habitants"

|Ň-tòbò| |bò-tòbò|

/PN1-action d'habiter/ /PN2-action d'habiter /

6) cl3/4 : ǹló "tête" / mìnló "têtes" 
Dans l'exemple 6, la nasale présente au singulier réapparait au pluriel. C'est elle qui pose des problèmes de découpage morphologique.

La nasale réapparait particulièrement dans certains nominaux de classe 4 , certains de classe 2 et de classe 6. Dans l'exemple 6, il s'agit d'un nominal de classe 4 . La question ici est de savoir si dans mìnló, la nasale après le préfixe nominal de classe 4 |mi-| est rattachable au thème, au préfixe nominal ou si c'est une reprise du préfixe nominal de classe 3.

\section{Quelques possibilités d'analyse}

17 Face au problème de la nasale syllabique évoqué plus haut, plusieurs possibilités de découpage sont envisageables.

\subsection{Hypothèse 1 : la double préfixation}

18 Pierre ONDO MEBIAME estime que le pluriel en fang ntúmú résulte d'une double préfixation. Autrement dit, pour lui, la nasale après les préfixes pluriel est une reprise du préfixe nominale singulier. Dans l'exemple 6, le découpage morphologique suggéré serait donc le suivant :

ǹló "tête" |Ǹ-ló|/PN3-tête/

mìnló "têtes" |mi-N-ló| /PN4-PN1-tête/.

19 La nasale initiale des nominaux problématiques joue effectivement le rôle de préfixe nominal. Elle est syllabique et porte un ton bas, sa réalisation est d'ailleurs assez proche d'une voyelle. En première écoute, on entendrait pratiquement un [ə] ([ə̀nló]). Au pluriel en revanche, la nasale n'est ni syllabique, ni porteuse d'un ton bas. Ceci signifie en principe que la nasale apparaissant dans la forme pluriel n'est pas une reprise du préfixe nominal singulier.

20 Si le préfixe nominal singulier était repris au pluriel, nous aurions une réalisation du type *miǹló, ce qui n'est pas le cas ici et n'est d'ailleurs pas admis en fang ntúmú.

\subsection{Hypothèse 2 : la nasale syllabique est rattachable au thème}

La nasale au pluriel n'étant pas une reprise du préfixe nominal singulier, MBA NKOGHE propose de la rattacher à la base. La consonne initial dans notre exemple serait donc une prénasalisée. Son point de vue suggèrerait le découpage suivant :

ǹló "tête" |Ǹ-nló| /PN3-tête/

mìnló "têtes" |mi-nló|/PN4-tête/.

Dans une langue proche, le mankon, LEROY qui observait un comportement similaire, proposait le même type d'approche. Pour elle, 'il est probable qu'à l'origine cette nasale était un préfixe nominal (et que le noyau constituait à lui seul la racine), mais elle ne peut plus être considérée comme tel actuellement puisqu'elle coexiste avec le préfixe de classe" . Autrement dit, la nasale est rattachable à la base. Mais, contrairement à MBA NKOGHE, elle postulera, dans certains cas, un morphème à signifiant zéro comme préfixe nominal singulier et reportera le ton sur la nasale au pluriel.

Exemple : 
[fintsáPá] fi-ǹ-tsáPá 'toupie” (cl.19)

[nì̀gòm] nì-̀̀-gòm "plantain" (cl.5)

La proposition faite par LEROY au sujet du mankon ne fonctionne pas en ntúmú. En mankon la nasale syllabique porteuse de ton se rattache à la base aussi bien lorsque le préfixe nominal est de type singulier que pluriel. En fang par contre cette nasale n'est jamais porteuse de ton au pluriel et n'accompagne aucun préfixe nominal singulier formellement marqué.

\subsection{Nouvelles considérations}

Nous avons vu plus haut que le fang ntúmú est à la fois tonale et accentuel. Nous constatons que les locuteurs marquent toujours une légère pause après le préfixe nominal. Ceci vaut aussi bien pour les nominaux posant un problème de découpage morphologique que pour ceux ayant une structure facilement décomposable.

Nous constatons en outre que la première syllabe de la base (thème ou racine verbonominale) est toujours accentuée. En fait pause et accent sont étroitement liés.

Exemples :

7) A'kó? "pierre" | à-kóP| /PN5-pierre/

Mə̀’kó? "pierres" | mò-kó?|/PN6-pierre/

8) ò'kòp "crochet" | ò-kòp|/PN11-crochet/

à'kòp "crochets" | à-kòp|/PN5-crochet/

En fang ntúmú, l'accent remplit donc une fonction démarcative en ce sens qu'il permet d'identifier non seulement la limite du préfixe nominal, mais également la première syllabe de la base.

En appliquant ce principe de découpage aux unités problématiques, on constate que la pause intervient après la nasale aussi bien au singulier qu'au pluriel. Le première syllabe dans les deux cas n'est pas réalisée pré nasalisée. Cette nouvelle considération sous-tend donc que la nasale au pluriel est rattachable au préfixe nominal et milite en faveur d'un préfixe nominal de structure CVN.

Dans l'exemple 6, la segmentation serait donc la suivante :

ǹló "tête" |N-ló|/PN3-tête/

mìnló "têtes" |min-ló| /PN4-tête/

9) m̀bò̀ "manioc" |N-bòn| /PN3-manioc/

mìmbòy "maniocs" |mìm-bòy| /PN4-manioc/

La nouvelle considération que nous venons de présenter permet d'avoir une construction du nominale unique : PN+BASE.

Parler d'une double préfixation aurait conduit à envisager deux types de constructions : il $y$ aurait d'une part la construction classique $\mathrm{PN}+\mathrm{BASE}$, et une construction $\mathrm{PN}+\mathrm{PN}+\mathrm{BASE}$ qui ne serait applicable qu'au nominaux ayant une nasale syllabique comme préfixe nominal singulier, c'est-à-dire une partie des nominaux de classe 2, et 4 . Ce qui semble peu économique.

\subsection{PN4 : Appariements et implications}

Un appariement est une mise en relation entre une classe singulier et son correspondant pluriel. Le fang Ntumu présente les appariements suivants : 
Cl.I

Cl.3 Cl. 4

Cl.5 Cl. 6

Cl.7 Cl. 8

Cl.9 Cl.2 Cl. 6

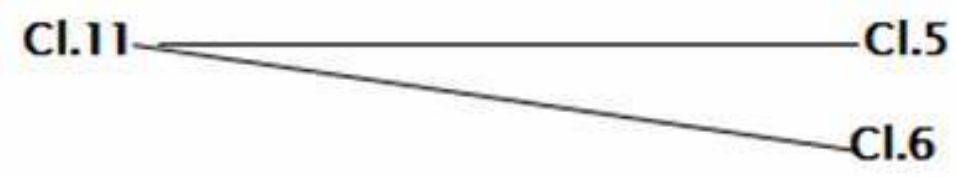

31

Le schéma ci-dessus montre qu'il n'existe qu'un appariement $\mathrm{Cl} 3 / \mathrm{Cl} 4$, les classes 3 et 4 ne pouvant s'apparier avec aucune autre classe. Ceci implique donc qu'en fang Ntumu, le PN4 ne présente qu'une forme unique: /miN-/, contrairement à la grande majorité des langues bantu dans laquelle il se présente majoritairement sous la forme /mi-/.

Pour les unités de classe 1, la nasale n'est pas systématiquement reprise au pluriel (classe2) comme le montre l'exemple 5. Ceci montre que la structure CVN n'est pas imputable à la présence d'une nasale syllabique dans la forme singulier, mais certainement à une construction propre aux unités de classe 4 .

\section{Conclusion}

Ce travail abordait l'une des questions fondamentales dans l'étude des langues bantu, à savoir la forme des indices de classe. Le problème de découpage morphologique des préfixes nominaux de classe 4 en fang ntúmú, et certainement dans d'autres langues bantu, est lié à la présence d'une nasale après le préfixe pluriel.

Nous avons vu que la pause et l'accent étaient déterminants dans l'identification des préfixes nominaux. En partant de ces deux paramètres, nous avons établi, contrairement à la tradition bantouiste, que le préfixe nominal des unités de classe 4 comportait non pas deux mais trois éléments, ce qui est tout-à-fait novateur. La formation du pluriel résulte donc, non pas d'une double préfixation, mais d'une structure préfixale complexe de type CVN. 


\section{BIBLIOGRAPHIE}

- IDIATA, D. F., LEITCH, M., REKANGA, J. P., ONDO MEBIAME, P., 2000, Les classes nominales et leur sémantisme dans les langues bantu du nord-ouest, Munich, Lincom Europa.

- KADIMA, M., 1969, Le système des classes en bantu, Leuven, vander.

- LEROY, J., 2007, Le mankon, langue bantu des Grassfields (province Nord-Ouest du Cameroun), Paris, Peeters.

- MBA-NKOGHE, J., 2001, Description du fang du Gabon (parler atsi), phonologie, morphologie, syntaxe, lexique, Thèse de Doctorat d'Etat ès-Lettres et sciences Humaines, Université de la Sorbonne Nouvelle-Paris III, Lille, A.N.R.T.

- MAHOO, 2008, NUGL Online, The online version of the New Updated Guthrie List, a referential classification of the Bantu languages, in http://goto.glocalnet.net/mahopapers/nuglonline.pdf, consulté le 09/01/2010.

\section{RÉSUMÉS}

Le fang est une langue bantu, il comporte cinq dialectes dont fait parti le ntúmú. Le fang ntúmú est une langue à classe. Tous les nominaux d'une classe gouvernent le même accord et la langue leur affecte un indice de type préfixal : le préfixe nominal.

Le préfixe nominal apporte des informations sur la classe, mais également sur le nombre. Les travaux menés au sujet du pluriel en fang ntúmú montrent que celui-ci est obtenu par l'agencement de deux préfixes : celui du pluriel et du singulier.

Nous montrerons pour notre part qu'il s'agit en réalité d'une construction préfixale complexe de type CVN, ce qui serait assez novateur puisque le préfixe nominal dans les langues bantu est généralement de type Ø-, C-, V- ou CV-. Notre communication s'inscrit dans le deuxième axe des RJC qui définit le cotexte comme l'ensemble des unités linguistiques.

Fang is a bantu language. It contains 5 dialects and ntúmú is one of them. Fang ntúmú makes classification of his nouns. All nouns of the same class govern the same agreement, and the language allocates to them a prefixal mark: the nominal prefix. The nominal prefix brings information not only about the class of every noun, but also about number. Some works about the plural in fang ntumu show that this one is obtained by the arrangement of two prefixes: that of plural and that of singular. We shall show for our part that the plural is really obtained by a complex prefixal construction with tree elements: a consonant, a vowel and a nasal. So, his structure is CVN. This conception is innovative because the structure of nominal prefix in bantu languages is generally, Ø-, C-, V-, or CV-. This communication joins in the second axis of RJC, which defines the cotext as all linguistic units.

\section{INDEX}

Mots-clés : accord, morphologie nominale, classe, pluriel, préfixe nominal, thème nominal

Keywords : agreement, nominal morphology, class, plural, nominal prefix, nominal stem, fang 
AUTEUR

RÉGIS OLLOMO ELLA

Université Paris 3 - Sorbonne Nouvelle 